\title{
GLI INSEGNAMENTI DELLA CRISI
}

\author{
ROBERTO ARTONI $(*)$
}

SuNTO. - La storia dimostra che le crisi economiche più profonde sono state innescate da tre fattori strettamente connessi: regolazione finanziaria carente, premessa per la deresponsabilizzazione degli intermediari finanziari; forte polarizzazione nella distribuzione del reddito associata ad elevato indebitamento delle famiglie; politiche economiche incapaci di mantenere la domanda aggregata ad un livello corrispondente ad un'adeguata utilizzazione delle risorse. I governi dovrebbero sia evitare il manifestarsi delle crisi, sia contrastarle con politiche appropriate.

$* * *$

ABSTRACT. - History shows that the major economic crises in the last century were triggered by three factors closely interconnected: financial deregulation, leading to irresponsibility of the intermediaries; strong polarization in the distribution of income usually associated with a huge rise in household debt; economic policies unable of keeping aggregate demand at a level corresponding to an adequate utilization of resources. Governments should both avoid the insurgence of the crises and solve them through suitable policies.

Nel mio intervento, per le mie limitate competenze, il tema delle banche sarà toccato solo tangenzialmente. A mia giustificazione, si deve sottolineare che le banche o gli intermediari finanziari sono una componente assolutamente importante del sistema economico e sociale e che devono pertanto essere inseriti in contesti più vasti, come tenterò di fare. Comincerò con alcuni riferimenti eterogenei che spero di riuscire a inquadrare nel corso dell'esposizione.

Il primo riferimento ha una dimensione storica o politica.

(*) Istituto Lombardo Accademia di Scienze e Lettere, Milano; Università Commerciale Luigi Bocconi, Milano, Italia. E-mail: roberto.artoni@unibocconi.it 
Winston Churchill ha sostenuto che la democrazia è la peggior forma di governo, eccezion fatta per tutte le altre forme finora sperimentate. Traducendo per i nostri fini, il sistema capitalistico è certamente il sistema più efficiente e meglio capace di soddisfare le esigenze non solo materiali della popolazione, se paragonato a tutte le alternative finora sperimentate.

Elaborando l'argomento, è tuttavia certo che non tutte le democrazie sono uguali, anche sotto il profilo strettamente economico. Alcuni regimi democratici sono meglio in grado di orientare il processo economico, attenuando gli elementi d'instabilità che hanno caratterizzato il mondo capitalistico negli ultimi due secoli.

Il secondo riferimento è più antico, risalendo al 500 a.C. e riguardando Menenio Agrippa. I plebei oppressi dai debiti si erano ritirati fuori dalla città, rifiutandosi di prestare ulteriormente la loro opera. Menenio Agrippa li convinse a rientrare con il famoso apologo: le membra si erano ribellate contro lo stomaco con il risultato che ben presto tutto il corpo era irrimediabilmente debilitato. Soltanto dalla concordia di tutte le componenti della società, o di tutti gli organi, poteva derivare il benessere generale. I plebei furono convinti dall'apologo e rientrarono in città ottenendo l'istituzione dei tribuni della plebe e la liberazione dai debiti.

Per i nostri fini tutto ciò può essere inteso come l'affermazione dell'esigenza di garantire un giusto equilibrio fra le diverse componenti dell'organizzazione sociale ed economica in ambito nazionale, o fra $\mathrm{i}$ diversi paesi in una struttura sovranazionale.

Quando accenneremo all'indebitamento delle famiglie e al connesso ruolo, oltre le righe in molto paesi, svolto dal sistema finanziario nel suo complesso, e lo considereremo come causa determinante delle crisi finanziarie dei sistemi capitalistici, un richiamo a Menenio Agrippa opportunamente riformulato non sarà superfluo.

Il terzo riferimento riguarda le politiche distributive. E' stato affermato che è interesse del singolo imprenditore pagare il meno possibile i suoi operai, ma è nell'interesse di quell'imprenditore che gli altri imprenditori paghino ai loro dipendenti salari per quanto possibile elevati.

Prescindendo dal fatto che alla base di questa affermazione sta una visione molto semplificata del processo produttivo che viene ad essere caratterizzato da un'estrema integrazione verticale, questa tesi indica che le problematiche distributive non possono essere confinate ad una dimensione microeconomica, ma devono essere opportunamente inqua- 
drate in un contesto più ampio, macroeconomico o lato sensu politico.

Sulla base di questi riferimenti possiamo tentare di interpretare le più importanti crisi che hanno investito i sistemi capitalistici negli ultimi 100 anni (nel 1929 e nel 2008).

Preliminarmente, si deve osservare che le due crisi hanno alcuni elementi comuni. Si è verificato un collasso finanziario all'esito di un lungo periodo di euforia, cui è seguita una forte contrazione nei livelli di attività, anche se questa contrazione è stata più forte negli anni '30 di quanto non è accaduto nell'ultimo decennio (soprattutto se si guarda agli Stati Uniti).

Richiamando alcuni elementi essenziali, l'euforia finanziaria degli anni '20 è stata caratterizzata da tre elementi:

- innovazioni finanziarie nella forma di investment trusts (dando nel medesimo tempo ampio spazio al finanziamento degli acquisti di borsa sulla base di margini molto ridotti);

- di conseguenza, forte ascesa delle quotazioni di borsa, anche in assenza di prospettive di reddito ragionevoli per molte imprese quotate;

- forte espansione del credito al consumo, destinato allora per buona parte all'acquisto di automobili.

Sono molte le analogie con le vicende che hanno preceduto la crisi del 2008. Le quotazioni azionarie sono cresciute fino a raggiungere il massimo nell'ottobre 2007, anche in questo caso sostenute da un quadro regolatorio molto permissivo e da un'articolata rete di intermediari finanziari.

La creazione di nuovi strumenti finanziari, che si pensava portasse ad un'opportuna distribuzione o frantumazione dei rischi, è proseguita senza soste.

L'indebitamento delle famiglie, sostenuto dall'illusione derivante dalla continua rivalutazione degli assets mobiliari e immobiliari, è cresciuto raggiungendo livelli storicamente molto elevati. Fra i molti, un dato può essere portato a sostegno delle precedenti affermazioni. Fra il 1980 e il 2005, per le famiglie degli Stati Uniti il rapporto fra l'ammontare del debito ipotecario e la mediana del reddito è passato dal 57 al $156 \%$. Riprendendo una tesi molto diffusa, si può ragionevolmente sostenere che le più importanti crisi finanziarie trovano alimento e sono state innescate, oltre che da una mediocre regolazione degli intermediari finanziari, da un forte indebitamento delle famiglie. Per l'ultima crisi ciò vale per gli Stati Uniti, ma anche in Europa per Spagna, Irlanda e Regno Unito. 
Deve essere comunque sottolineato che questo indebitamento è il riflesso o l'effetto di un processo di forte concentrazione della ricchezza. Anche in questo caso possiamo fornire alcuni dati. In USA nel periodo 1993-2012 il reddito medio pro capite in termini reali è cresciuto negli Stati Uniti del 18\%; il top 1\% dei redditieri ha visto crescere il suo reddito pro capite dell' $86 \%$; il restante $99 \%$ del 6,6\%. Di fatto il $68 \%$ della crescita è andato al top $1 \%$. In altri termini, i benefici della crescita degli anni 1993-2012 sono andati ad una porzione molto ridotta della popolazione.

Possiamo accennare anche alla destinazione dell'indebitamento. Se negli anni '20 era destinato all'acquisto di beni durevoli, negli anni più recenti sembra essere stato destinato in larga misura all'acquisto della casa, oltre che all'istruzione e alle carte di credito. Tutto ciò è sia il riflesso della polarizzazione nella distribuzione del reddito, sia dell'assenza dell'intervento pubblico nella fornitura di alcuni servizi fondamentali.

Possiamo a questo punto tornare a due dei precedenti riferimenti. Per Menenio Agrippa, a tutti gli organi del corpo umano sono affidati specifici compiti e conseguenze nefaste si manifestano quando le attribuzioni non sono rispettate. In altri termini, si può anche sostenere che i danni si manifestano quando un organo assume funzioni che non gli competono, allargando la sfera delle sue attività. Nel nostro caso si può ritenere che quando gli intermediari finanziari si sostituiscono alla collettività nella fornitura di servizi, meritevoli ed essenziali, si mettono in moto meccanismi alla lunga autodistruttivi, che nel caso specifico hanno colpito anche le classi medie. Tutti eravamo compiaciuti per la crescita relativamente più elevata degli Stati Uniti nel decennio pre2008, ma questo differenziale di crescita derivava per larga parte dall'esplosione del debito privato. Il compiacimento europeo era alimentato in particolare dal disavanzo commerciale USA che consentiva di sostenere le esportazioni europee e asiatiche.

L'esperienza recente è significativa anche per le problematiche distributive. Le potenzialità produttive di un paese possono trovare realizzazione se alla loro espansione corrisponde un'adeguata domanda: le fonti di questa domanda possono derivare o dall'estero (ma non tutti i paesi possono essere in surplus; almeno uno deve essere in deficit, e non può esserlo in eterno).

In alternativa la domanda aggregata può essere sostenuta dall'interno, in una prima ipotesi come effetto di politiche monetarie molto 
permissive (com'è di fatto accaduto prima e anche dopo la crisi). Quest'ultimo meccanismo, apparentemente indolore, non può essere protratto all'infinito, come testimoniano le vicende prima ricordate.

Al fine del sostegno della domanda, le alternative fisiologiche sono costituite o da politiche pubbliche (anche queste non estendibili oltre certi limiti) o dall'attivazione di meccanismi istituzionali che, come già detto, hanno il fine essenziale di garantire un'equilibrata distribuzione del reddito fra tutte le fasce della popolazione.

Questo è l'insegnamento fondamentale del New Deal rooseveltiano caratterizzato da politiche innovative nella sfera sociale e nelle opere pubbliche, oltre che nella sfera della contrattazioni salariale. Non sembra che le politiche degli ultimi decenni abbiano operato in questo senso.

A questo punto possiamo infatti affrontare l'ultimo argomento riguardante i diversi effetti delle due crisi sui livelli di attività, sul prodotto interno lordo.

L'esperienza degli anni '30, anche alla luce delle teorie keynesiane, aveva dimostrato che era eccessiva la fiducia nella capacità dei mercati di portare a soluzioni ottimali. Si può qui tornare a Churchill, con il riconoscimento che i sistemi capitalistici possono essere diversamente articolati e quindi più o meno funzionali.

Dall'analisi dell'effettivo funzionamento dei sistemi capitalistici è derivata nel secondo dopoguerra la creazione o l'attivazione di alcuni istituti (stato sociale, contrattazione collettiva, imprese pubbliche in diversa forma) che avevano o dovrebbero ancora avere il fine di garantire una distribuzione del reddito equilibrata, oltre che di stabilizzare l'evoluzione ciclica dei sistemi capitalistici.

A ciò si aggiunga che il sistema monetario internazionale, come era stato organizzato a seguito degli accordi di Bretton Woods, tendeva a regolare i movimenti internazionali di capitale, scoraggiando i comportamenti speculativi. In questo quadro i saldi del bilancio pubblico dovevano essere collocati nel più generale andamento macroeconomico: il pareggio del bilancio, almeno nella parte corrente, doveva essere perseguito in un contesto di adeguato utilizzo della capacità produttiva. Questi istituti o princìpi hanno guidato il mondo capitalistico per tre decenni con brillanti risultati.

L'adesione a questi princìpi, anche per i fenomeni inflazionistici connessi al crollo di Bretton Woods, è venuta meno a partire dal 1980, quando è stata adottata una politica economica che si è posta in sostanziale contrapposizione a quella seguita nei decenni precedenti, sia sul 
piano degli assetti economici e sociali dei principali paesi capitalistici, sia nella sfera dei rapporti finanziari internazionali caratterizzati negli ultimi decenni da una piena libertà di movimento dei capitali.

Ma la storia non raggiunge mai un punto di quiete. Qui possiamo solo osservare che la crisi del 2007 con le sue conseguenze ha riproposto la contrapposizione fra due modelli di politica economica, la prima in linea con quella degli anni '80 e degli anni '20 del secolo scorso e la seconda vicina a quella del secondo dopoguerra.

In Europa, su impulso del paese dominante sul piano economico ed esportatore netto di merci, oltre che finanziatore di molti paesi dell'Unione Europea, la Germania, si è affermata la cosiddetta austerità: ricerca della competitività attraverso la compressione salariale e controllo stretto dei disavanzi pubblici a prescindere dalle condizioni macroeonomiche. I risultati non sono oggettivamente esaltanti, pur essendo stata seguita una politica monetaria certamente eterodossa e molto permissiva.

Negli Stati Uniti si è affermata una linea molto più flessibile (sostenuta anche sul piano accademico da un revival post keynesiano) che ha dato luogo a risultati per molti versi soddisfacenti, anche se non spettacolari, in termini di occupazione e di crescita economica. Ritornando a Churchill, come non tutte le democrazie sono uguali, così non tutti i sistemi capitalistici o non tutte le politiche economiche sono uguali.

Non spetta a me indicare quale è la via migliore per ottenere risultati positivi nel lungo periodo, anche se gli insegnamenti degli ultimi anni, pur ambigui sotto molti aspetti, sembrano essere univoci. 